\title{
Mathematical analysis of approximate biological effective dose (BED) calculation for multi-phase radiotherapy treatment plans
}

\author{
Kevin I Kauweloa, Alonso N Gutierrez, Angelo Bergamo, Sotirios Stathakis, \\ Niko Papanikolaou, Panayiotis Mavroidis \\ Department of Radiation Oncology, University of Texas Health Science Center at San Antonio, San Antonio, TX, USA. \\ Cancer Therapy and Research Center, San Antonio, TX, USA.
}

Received March 19, 2014; Published Online April 08, 2014

[Presented at the Young Investigator's Symposium at the 2014 Annual Meeting of

Southwest Chapter of American Association of Physicists in Medicine (AAPM) in San Antonio, Texas, USA]

\section{Conference Proceeding}

\begin{abstract}
Purpose: There is growing interest about biological effective dose (BED) and its application in treatment plan evaluation due to its stronger correlation with treatment outcome. An approximate biological effective dose $\left(B E D_{A}\right)$ equation was introduced in order to simplify BED calculations by treatment planning systems in multi-phase treatments. The purpose of this work is to reveal its mathematical properties relative to the true, multi-phase $\mathrm{BED}$ (ВEDT) equation.
\end{abstract}

Methods: The BEDT equation was derived and used to reveal the mathematical properties of BEDA. MATLAB (MathWorks, Natick, MA) was used to simulate and analyze common and extreme clinical multi-phase cases. In those cases, percent error and Bland-Altman analysis were used to study the significance of the inaccuracies of BEDA for different combinations of total doses, numbers of fractions, doses per fractions and $\alpha / \beta$ values. All the calculations were performed on a voxel-basis in order to study how dose distributions would affect the accuracy of BEDA.

Results: When the voxel dose-per-fractions (DPF) delivered by both phases are equal, BEDA and BEDт are equal $(0 \%$ error). In heterogeneous dose distributions, which significantly vary between the phases, there are fewer occurrences of

Presenting author: Kevin Kauweloa; Department of Radiation Oncology, University of Texas Health Science Center at San Antonio, San Antonio, TX, USA.

Cite this article as:

Kauweloa KI, Gutierrez AN, Bergamo A, Stathakis S, Papanikolaou N, Mavroidis P. Mathematical analysis of approximate biological effective dose (BED) calculation for multi-phase radiotherapy treatment plans. Int J Cancer Ther Oncol 2014;

2(2):020226. DOI: $10.14319 /$ ijcto.0202.26 equal DPFs and hence the imprecision of BEDA is greater. It was shown that as the $\alpha / \beta$ ratio increased the accuracy of $\mathrm{BED}_{\mathrm{A}}$ would improve. Examining twenty-four cases, it was shown that the range of DPF ratios for 3\% Perror varied from 0.32 to $7.50 \mathrm{~Gy}$, whereas for $\mathrm{P}$ error of $1 \%$ the range varied from 0.50 to $2.96 \mathrm{~Gy}$.

Conclusion: The DPF between the different phases should be equal in order to render BEDA accurate. OARs typically receive heterogeneous dose distributions hence the probability of equal DPFs is low. Consequently, the BEDA equation should only be used for targets or OARs that receive uniform or very similar dose distributions by the different treatment phases.

Impact: To our knowledge, it is a usual practice to use $\mathrm{BED}_{\mathrm{A}}$ instead of $\mathrm{BED}_{\mathrm{T}}$ due to its convenience with current treatment planning systems (TPS). This was shown to be a potential problem with our mathematical analysis of BEDA. The goal of this work was to quantitatively measure the accuracy of $\mathrm{BED}_{\mathrm{A}}$ under many different circumstances, and also reveal its potential imprecision in clinical circumstances regarding healthy organs. This work puts emphasis on the importance of creating future TPS with the capability of calculating the $\mathrm{BED}_{\mathrm{T}}$.

Key Results: Using the multi-phase equations of $\mathrm{BED}_{\mathrm{T}}$ and $\mathrm{BED}_{\mathrm{A}}$, specifically two-phases (e.g., primary and boost), the percent error $\left(\mathrm{P}_{\text {error }}\right)$ equation was derived. Five variables within this equation are the $\alpha / \beta$ ratio, which is a tissue-dependent constant of the linear-quadratic (LQ) cell- survival curve model, 
the dose-per-fractions (DPF) for both phases, and the number of fractions for both phases. The $\mathrm{P}_{\text {error }}$ equation reveals the many varying factors in the accuracy of $\mathrm{BED}_{\mathrm{A}}$. Figure 1 displays the change of $\mathrm{P}_{\text {error }}$ with respect to four of the variables, except $\alpha / \beta$, which is instead shown in Figure 2. As seen, the main determining factor in the accuracy of $\mathrm{BED}_{\mathrm{A}}$ is the DPF-ratio, which, when it gets equal to $1, \mathrm{P}_{\text {error }}$ becomes equal to $0 \%$ (see Figure 1). Since DPF- ratio is the most important factor in the accuracy of $\mathrm{BED}_{\mathrm{A}}$, and due to its dependence upon the dose distribution, we have shown that using $\mathrm{BED}_{\mathrm{A}}$ on OARs could potentially lead to significant errors. On the other hand, its application to target volumes would instead produce less error due to fact that target volumes typically receive more homogeneous dose distributions.
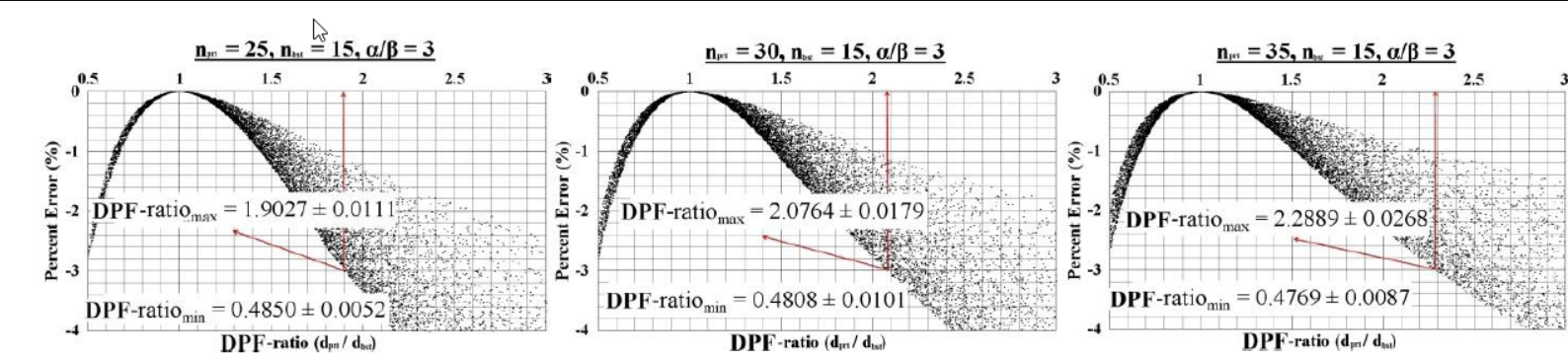

FIG. 1: As seen in all three plots, as the DPF-ratio steers from 1, $\mathrm{P}_{\text {error }}$ increases. Also, as $\mathrm{n}_{\text {pri }} / \mathrm{n}_{\text {bst }}$ increases, $\mathrm{P}_{\text {error }}$ decreases. The DPF-ratio ${ }_{\max }$ and DPF-ratio $o_{\min }$ reveal the ratios that lead to a $3 \%$ error of $\mathrm{BED}_{\mathrm{A}}$.
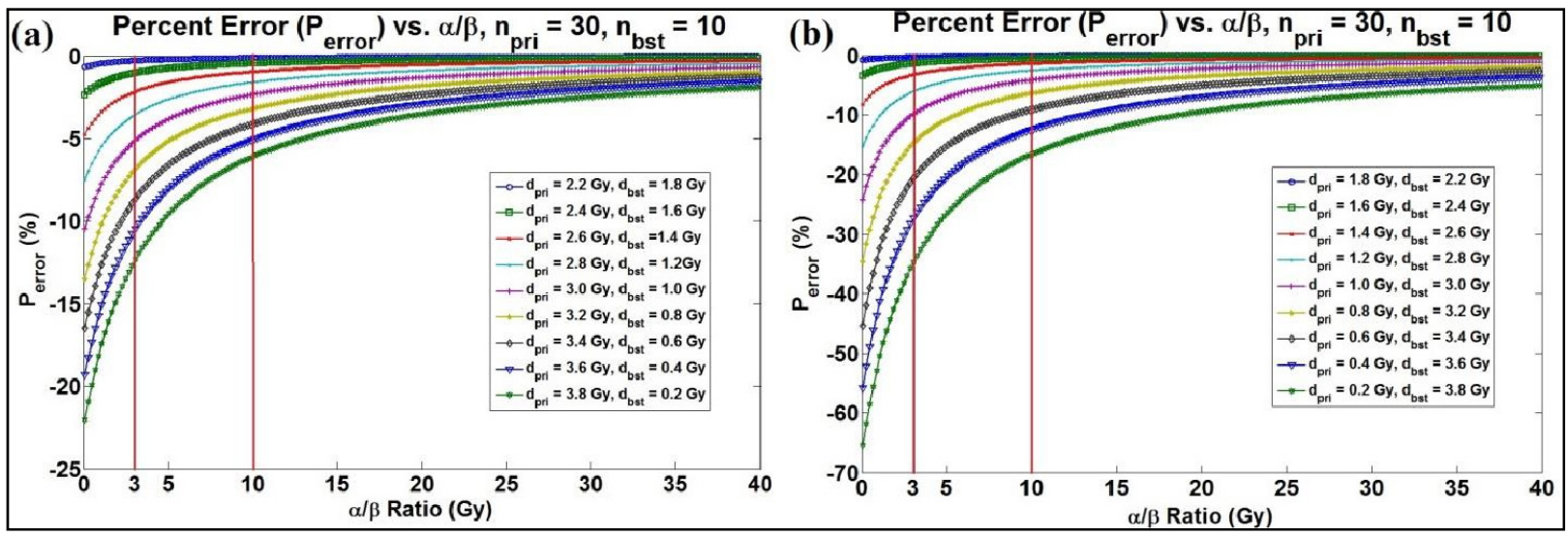

FIG. 2: (a) This is a plot with DPF-ratios $>1$, while (b) is with DPF-ratios $<1$. When $d_{\text {pri }} / d_{\text {bst }}$ is less than one, notice the steeper drop in $P_{\text {error }}$ with a change in DPF. 